\title{
A new rapid method for direct antimicrobial susceptibility testing of bacteria from positive blood cultures
}

\author{
Simona Barnini ${ }^{1}$, Veronica Brucculeri ${ }^{1}$, Paola Morici ${ }^{2}$, Emilia Ghelardi ${ }^{2}$, Walter Florio ${ }^{2}$ and Antonella Lupetti ${ }^{2}$
}

\begin{abstract}
Background: Rapid identification and antimicrobial susceptibility testing (AST) of the causative agent(s) of bloodstream infections can lead to prompt appropriate antimicrobial therapy. To shorten species identification, in this study bacteria were recovered from monomicrobial blood cultures by serum separator tubes and spotted onto the target plate for direct MALDI-TOF MS identification. Proper antibiotics were selected for direct AST based on species identification. In order to obtain rapid AST results, bacteria were recovered from positive blood cultures by two different protocols: by serum separator tubes (further referred to as PR1), or after a short-term subculture in liquid medium (further referred to as PR2). The results were compared with those obtained by the method currently used in our laboratory consisting in identification by MALDI-TOF and AST by Vitek 2 or Sensititre on isolated colonies.

Results: The direct MALDI-TOF method concordantly identified with the current method $97.5 \%$ of the Gram-negative bacteria and $96.1 \%$ of the Gram-positive cocci contained in monomicrobial blood cultures. The direct AST by PR1 and PR2 for all isolate/antimicrobial agent combinations was concordant/correct with the current method for 87.8 and 90. $5 \%$ of Gram-negative bacteria and for 93.1 and $93.8 \%$ of Gram-positive cocci, respectively. In particular, $100 \%$ categorical agreement was found with levofloxacin for Enterobacteriaceae by both PR1 and PR2, and 99.0 and $100 \%$ categorical agreement was observed with linezolid for Gram-positive cocci by PR1 and PR2, respectively. There was no significant difference in accuracy between PR1 and PR2 for Gram-negative bacteria and Gram-positive cocci.
\end{abstract}

Conclusions: This newly described method seems promising for providing accurate AST results. Most importantly, these results would be available in a few hours from blood culture positivity, which would help clinicians to promptly confirm or streamline an effective antibiotic therapy in patients with bloodstream infections.

Keywords: Bloodstream infection, Blood culture, Direct AST, Bactec FX, Alfred 60AST, MALDI-TOF

\section{Background}

Rapid identification (ID) and antimicrobial susceptibility testing (AST) of the causative agent(s) of bloodstream infections (BSI) are essential for the timely selection of an appropriate antimicrobial therapy, which may result in a better outcome for patients [1-4], and contribute to contrast the emergence of antimicrobial resistance [5].

The current method requires that a positive blood culture $(\mathrm{BC})$ is subcultured onto solid media before ID and AST are performed by automated systems. To shorten

\footnotetext{
* Correspondence: antonella.lupetti@med.unipi.it

${ }^{2}$ Dipartimento di Ricerca Traslazionale e delle Nuove Tecnologie in Medicina

e Chirurgia, Università di Pisa, Pisa, Italy

Full list of author information is available at the end of the article
}

the turnaround time for diagnosis, several methods have been proposed and evaluated, some of which with promising results [6-21].

In the present study, we aimed at establishing a method able to provide rapid AST results, further referred to as direct AST. To this aim, we used a CE approved system, named Alfred 60AST (Alifax), initially conceived for urine screening, and for AST of bacterial isolates from urine [22]. In order to adapt the system to rapid AST from positive blood cultures, two different protocols, protocol 1 (PR1) and protocol 2 (PR2), were evaluated, the first using bacteria harvested from positive $\mathrm{BC}$ by serum separator tubes, the second using a short-term subculture of a positive BC in liquid medium. MALDI- 
TOF identification of bacteria contained in monomicrobial BC was performed by a recently described method, further referred to as direct MALDI-TOF method [11]. The ID results obtained by the direct MALDI-TOF method and the AST results obtained by PR1 and PR2 were compared with those by the method currently used in our laboratory, further referred to as current method, i.e. ID and AST on isolated colonies by MALDI-TOF and Vitek 2 or Sensititre, respectively.

\section{Methods}

\section{Blood samples}

Blood specimens from patients admitted to the Pisa University Hospital (Italy) in the period July-December 2014 were inoculated into $\mathrm{BC}$ bottles [Plus Aerobic/F and Plus Anaerobic/F, or Peds Plus F (Becton Dickinson \& Co, BD, Milan, Italy)], collected at the Unità Operativa di Microbiologia, and transferred to the Bactec FX instrument (Becton Dickinson, Franklin Lakes, NJ, USA) for monitoring bacterial growth. From each patient, only the first positive $\mathrm{BC}$ apparently monomicrobial at the Gram staining was included in this study.

\section{Identification and AST of bacteria by the current method}

$\mathrm{BC}$ positive at the Bactec FX instrument were subjected to Gram staining and subcultured onto appropriate solid media. Isolated colonies were identified by MALDI-TOF (Bruker Daltonics, Bremen, Germany) and AST was performed by Vitek 2 (advanced Expert System software, version R04.02C; bioMérieux, Marcy l'Étoile, France) or, for Acinetobacter baumannii and Stenotrophomonas maltophilia isolates, by the Sensititre Aris system (Trek Diagnostic Systems, Thermo Fisher Scientific, OH, USA). The ID and AST results by the current method were used as comparator for evaluation of results obtained by PR1 and PR2.

Identification and AST cards were tested periodically for quality control assessment.

\section{Identification of bacteria by the direct MALDI-TOF method}

Identification of bacteria by the direct MALDI-TOF method was performed as previously described [11]. MALDI-TOF analysis was performed using a Microflex LT system mass spectrometer following the manufacturer's instructions. Captured spectra were analyzed using a MALDI-TOF Biotyper automated control and the Bruker Biotyper 3.1 software and library (4624 isolates) (Bruker Daltonics). For each plate, the instrument was calibrated to validate the run.

Identification results by the direct MALDI-TOF method were compared with those by MALDI-TOF on isolated colonies, as reference. Discrepancies in identification were resolved by Vitek 2 .

\section{Direct AST of bacteria}

Direct AST of bacteria was performed by Alfred 60AST (Alifax SpA, Polverara, PD, Italy) and carried out using two different protocols, PR1 and PR2. By PR1, bacteria were recovered from positive $\mathrm{BC}$ by serum separator tubes (BD) as previously described [11]. Briefly, an eight$\mathrm{ml}$ sample of a positive blood culture was transferred to serum separator tubes. Next, bacteria were sedimented on the surface of the silicon layer of the vacutainer tube by centrifugation at $2,000 \times \mathrm{g}$ for $10 \mathrm{~min}$, and suspended at 0.9 McFarland in vials containing $2 \mathrm{ml} \mathrm{HB \& L}$ broth (Alifax). The bacterial suspension was transferred into an AST-Empty vial and loaded in the refrigerated area dedicated to external samples, called "External Samples Zone" of the Alfred 60AST system. By PR2, $20 \mu \mathrm{l}$ of positive $\mathrm{BC}$ were inoculated into $2 \mathrm{ml} \mathrm{HB \& L}$ broth and loaded in the thermostat of the Alfred 60AST system for monitoring the bacterial growth up to 0.5 McFarland. Next, the instrument automatically transferred the sample into an AST-Empty vial, placed in the refrigerated area "Alfred Sample Zone". The time needed to reach a bacterial density corresponding to 0.5 McFarland was $1-4 \mathrm{~h}$, depending on the bacterial species ( $6 \mathrm{~h}$ for S. maltophilia).

Lyophilized antibiotics (Alifax) were dissolved in $2 \mathrm{ml}$ regenerating solution, and stored at $4{ }^{\circ} \mathrm{C}$ for up to 6 days (3 days for meropenem). The regenerated antibiotics were loaded into the "Alfred Antibiotic Zone". The antibiotics tested were: levofloxacin and gentamicin for all Gram-negative bacteria; cefotaxime, ceftazidime and meropenem for Enterobacteriaceae, and amikacin and colistin for nonfermenters; linezolid and teicoplanin for all Grampositive cocci; cefoxitin for staphylococci, and ampicillin for streptococci.

For each strain a panel of antibiotics was established on the basis of antibiotics available for this system at the time we started this study, and on the basis of the time needed to perform the test. We preferably selected antibiotics requiring three hours incubation instead of five. Each antibiotic was $C E$ approved. The vials, each containing $2 \mathrm{ml}$ HB\&L broth, were put in the thermostat, and automatically loaded with the bacterial suspension $(100 \mu \mathrm{l})$ and the selected antibiotic $(200 \mu \mathrm{l})$ for both PR1 and PR2. One vial was loaded only with the bacterial suspension and used as a positive control for bacterial growth.

\section{Interpretation of AST results and data analyses}

The antimicrobial activity of the tested antibiotics (by PR1 and PR2) was calculated by the system as percentage of bacterial growth in comparison to the positive control. The percentage of bacterial growth was automatically translated into clinical categories: a growth between 100 and $65 \%$ was interpreted as resistant, between 65 and $50 \%$ as intermediate, and $<50 \%$ as susceptible (EUCAST-defined breakpoints [23]). The clinical 
categories obtained by the different methods were compared with the results by the current method and expressed as: agreement, very major errors (false susceptible), major errors (false resistant), and minor errors (susceptible/resistant versus intermediate susceptibility). Discrepancies between the results by direct AST (PR1 or PR2) and the current method were resolved by Etest ( $A B$ BioMerieux), which was performed according to the manufacturer's instructions.

The percentages of very major errors and major errors were calculated according to the current ISO 20776-2 guideline. Major error rate was calculated based on the number of susceptible strains tested as denominator; very major error rate was calculated based on the number of resistant strains tested as denominator.

Statistical analysis was performed using the chi-square test for independent pairs. The level of significance was set at $P<0.05$.

\section{Results \\ Identification of bacteria from positive $\mathrm{BC}$ by direct MALDI-TOF}

A total of 194 positive $\mathrm{BC}$ were apparently monomicrobial at the Gram staining, eleven (5.7\%) of which resulted to be polymicrobial after subculture and were excluded from this study. All monomicrobial BC contained either Gramnegative bacilli or Gram-positive cocci. Eighty monomicrobial BC containing Gram-negative bacteria and 103 containing Gram-positive cocci were processed by the direct MALDI-TOF method, with a mean identification time of 25 min after BC positivity. In parallel, BC were subcultured onto agar media for microbial identification by the current method.
Identification of Gram-negative bacteria by the direct MALDI-TOF method gave an interpretable result for 78 (97.5\%) of 80 BC (Table 1), 73 (91.3\%) of which with scores $>2.0$. The unidentified strains were correctly identified by MALDI-TOF the day after on isolated colonies. Duplicates were $100 \%$ concordant and gave similar scores. No strain was misidentified by the direct MALDI-TOF method. Identification of bacteria by the direct and current methods by MALDI-TOF was always concordant.

Identification of Gram-positive cocci by the direct and current methods by MALDI-TOF gave concordant results for $99(96.1 \%)$ of the $103 \mathrm{BC}$, although only 45 (43.7\%) with scores $\geq 2.0$ (Table 2). Duplicates were $100 \%$ concordant and gave similar scores.

One Staphylococcus hominis was erroneously identified by the direct method as Staphylococcus haemolyticus, and one Streptococcus pneumoniae as Streptococcus oralis group mitis. Furthermore, one $S$. hominis and one Staphylococcus aureus strains were unidentified by the direct method and correctly identified by MALDI-TOF on isolated colonies.

\section{Direct AST of Gram-negative bacilli}

The AST results by PR1 and/or PR2 were available for all the 80 Gram-negative isolates tested, 62 of which were Enterobacteriaceae (Table 3) and 18 nonfermenters (Table 4). The number of resistant, intermediate, and sensitive strains for each antibiotic according to the current method is reported as additional material in (Additional file 1: Table S1). The AST by PR1 failed for 6 isolates (1 Enterobacter cloacae, 2 P. aeruginosa, and $3 A$. baumannii), and by PR2 for other 6 (1 K. pneumoniae, 1

Table 1 Gram-negative bacteria from monomicrobial blood cultures identified by the direct MALDI-TOF method

\begin{tabular}{|c|c|c|c|c|c|c|}
\hline \multirow[t]{2}{*}{ Species } & \multicolumn{4}{|c|}{ Correctly identified with score value of: } & \multirow[t]{2}{*}{ Unidentified } & \multirow[t]{2}{*}{ Total } \\
\hline & $3.000-2.300$ & $2.299-2.000$ & $1.999-1.700$ & $<1.700$ & & \\
\hline Escherichia coli & 17 & 7 & & & & 24 \\
\hline Klebsiella pneumoniae & 12 & 11 & 1 & & 1 & 25 \\
\hline Enterobacter aerogenes & 1 & 1 & & & & 2 \\
\hline Enterobacter cloacae & & 4 & & & & 4 \\
\hline Morganella morganii & 1 & 1 & & & & 2 \\
\hline Serratia marcescens & & 1 & 1 & & & 2 \\
\hline Salmonella spp. & & 1 & & & & 1 \\
\hline Proteus mirabilis & 1 & & & & & 1 \\
\hline Raoultella ornithinolytica & 1 & & & & & 1 \\
\hline Pseudomonas aeruginosa & 5 & 3 & 2 & 1 & 1 & 12 \\
\hline Acinetobacter baumannii & & 3 & & & & 3 \\
\hline Stenotrophomonas maltophilia & & 2 & & & & 2 \\
\hline Acinetobacter ursingii & & 1 & & & & 1 \\
\hline Total & 38 & 35 & 4 & 1 & 2 & 80 \\
\hline
\end{tabular}


Table 2 Gram-positive cocci from monomicrobial blood cultures identified by the direct MALDI-TOF method

\begin{tabular}{|c|c|c|c|c|c|c|c|}
\hline \multirow[t]{2}{*}{ Species } & \multicolumn{4}{|c|}{ Correctly identified with score value of: } & \multirow[t]{2}{*}{ Unidentified } & \multirow[t]{2}{*}{ Misidentified } & \multirow[t]{2}{*}{ Total } \\
\hline & $3.000-2.300$ & $2.299-2.000$ & $1.999-1.700$ & $<1.700$ & & & \\
\hline Staphylococcus epidermidis & & 8 & 21 & 13 & & & 42 \\
\hline Staphylococcus aureus & & 12 & 3 & 1 & 1 & & 17 \\
\hline Staphylococcus hominis & & 5 & 4 & 2 & 1 & 1 & 13 \\
\hline Staphylococcus capitis & 1 & 9 & 1 & & & & 11 \\
\hline Staphylococcus haemolyticus & & 4 & & 1 & & & 5 \\
\hline Staphylococcus warneri & & & 1 & 1 & & & 2 \\
\hline Staphylococcus pettenkoferi & & & 1 & & & & 1 \\
\hline Staphylococcus sciuri & & 1 & & & & & 1 \\
\hline Staphylococcus lugdunensis & & & 1 & & & & 1 \\
\hline Enterococcus faecium & 1 & 2 & 1 & & & & 4 \\
\hline Enterococcus faecalis & 1 & 1 & 1 & & & & 3 \\
\hline Enterococcus casseliflavus & & & 1 & & & & 1 \\
\hline Streptococcus sanguinis & & & & 1 & & & 1 \\
\hline Streptococcus pneumoniae & & & & & & 1 & 1 \\
\hline Total & 3 & 42 & 35 & 19 & 2 & 2 & 103 \\
\hline
\end{tabular}

Serratia marcescens, 1 Acinetobacter ursingii, $1 P$. aeruginosa, and 2 S. maltophilia). Overall, the antimicrobial susceptibility testing of Gram-negative bacilli (Tables 3 and 4) was assessed for a total of 353 (PR1) and 348 (PR2) isolate/antimicrobial agent combinations. Discrepancies between the direct and current methods were resolved by Etest, which revealed that AST was correct by PR1 or PR2, but not by the current method, for 15 (4.2) and 9 (2.6\%) isolate/antimicrobial agent combinations, respectively, all of which, but one, regarding
Enterobacteriaceae. Complete AST agreement (i.e., AST agreement for all tested antibiotics) was found for 35 (43.7) and 38 (47.5 \%) out of the 80 isolates by PR1 and PR2, respectively. The AST results of Gram-negative bacilli and the total percent error as well as the percent error for each antimicrobial agent are shown in Tables 3 and 4 . Together, concordant/correct AST for Gram-negative bacteria was found for 87.8 and $90.5 \%$ of all isolate/antimicrobial combinations by PR1 and PR2, respectively. Notably, AST agreement was higher than $90 \%$ for cefotaxime,

Table 3 Antimicrobial susceptibility testing of Enterobacteriaceae from positive blood cultures by PR1 (A) and PR2 (B) ${ }^{a}$

\begin{tabular}{|c|c|c|c|c|c|}
\hline Antimicrobial agent & No. of very major errors & No. of major errors & No. of minor errors & AST agreement & Total \\
\hline \multicolumn{6}{|l|}{$\bar{A}$} \\
\hline Cefotaxime (CTX) & $0 / 40$ & $1 / 19(5.3 \%)$ & $2 / 59(3.4 \%)$ & 56 (94.9\%) & 59 \\
\hline Ceftazidime (CAZ) & $0 / 32$ & $10 / 25(40 \%)$ & $4 / 60(6.7 \%)$ & $46(76.7 \%)$ & 60 \\
\hline Gentamicin (GM) & $0 / 24$ & 10/35 (28.6 \%) & $2 / 61(3.3 \%)$ & 49 (80.3\%) & 61 \\
\hline Levofloxacin (LEV) & $0 / 34$ & $0 / 26(0 \%)$ & $0 / 60$ & $60(100 \%)$ & 60 \\
\hline Meropenem (MEM) & $0 / 18$ & $5 / 43(11.6 \%)$ & $2 / 61(3.3 \%)$ & $54(88.5 \%)$ & 61 \\
\hline Total (\%) & $0 / 148$ & 26/148 (17.6 \%) & 10/301 (3.3 \%) & $265(88.1 \%)$ & 301 \\
\hline \multicolumn{6}{|l|}{ B } \\
\hline Cefotaxime (CTX) & 6/39 (15.4\%) & $0 / 19$ & $0 / 58$ & 52 (89.7 \%) & 58 \\
\hline Ceftazidime (CAZ) & $0 / 31$ & $1 / 24(4.2 \%)$ & $3 / 58(5.2 \%)$ & 54 (93.1 \%) & 58 \\
\hline Gentamicin (GM) & $0 / 25$ & 8/33 (24.2 \%) & $1 / 60(1.7 \%)$ & $51(85 \%)$ & 60 \\
\hline Levofloxacin (LEV) & $0 / 34$ & $0 / 24$ & $0 / 58$ & $58(100 \%)$ & 58 \\
\hline Meropenem (MEM) & $0 / 16$ & 6/42 (14.3\%) & $2 / 58(3.5 \%)$ & 50 (86.2 \%) & 58 \\
\hline Total (\%) & $6 / 145(4.1 \%)$ & 15/142 (10.6 \%) & 6/292 (2.1\%) & $265(90.7 \%)$ & 292 \\
\hline
\end{tabular}

a Using the results from the susceptibility testing of the bacteria by Vitek 2 as reference. In case of discrepancies between the two methods, the results for the susceptibility testing were confirmed by E-test. Data are numbers (with percentages) of bacterial isolates for which the antimicrobial susceptibility testing was concordant/correct or erroneous by PR1 (A) or PR2 (B) 
Table 4 Antimicrobial susceptibility testing of Gram-negative nonfermenters from positive blood cultures by PR1 (A) or PR2 (B) ${ }^{a}$

\begin{tabular}{|c|c|c|c|c|c|}
\hline Antimicrobial agent & No. of very major errors & No. of major errors & No. of minor errors & AST agreement & Total \\
\hline \multicolumn{6}{|l|}{ A } \\
\hline Amikacin (AMK) & $0 / 3$ & $0 / 10$ & $0 / 13$ & $13(100 \%)$ & 13 \\
\hline Colistin (COL) & $1 / 1(100 \%)$ & $1 / 12(8.3 \%)$ & $0 / 13$ & 11 (84.6 \%) & 13 \\
\hline Gentamicin (GM) & $1 / 2(50 \%)$ & $1 / 11(9.1 \%)$ & $0 / 13$ & $11(84.6 \%)$ & 13 \\
\hline Levofloxacin (LEV) & 1/9 (11.1\%) & $0 / 4$ & 2/13 (15.4\%) & 10 (76.9 \%) & 13 \\
\hline Total (\%) & $3 / 15(20 \%)$ & $2 / 37(5.4 \%)$ & $2 / 52(3.8 \%)$ & 45 (86.5 \%) & 52 \\
\hline \multicolumn{6}{|l|}{ B } \\
\hline Amikacin (AMK) & $0 / 4$ & $0 / 10$ & 0 & $14(100 \%)$ & 14 \\
\hline Colistin (COL) & $0 / 0$ & $0 / 14$ & 0 & $14(100 \%)$ & 14 \\
\hline Gentamicin (GM) & $1 / 3(33.3 \%)$ & 2/11 (18.2\%) & $1 / 14(7.1 \%)$ & 10 (71.5 \%) & 14 \\
\hline Levofloxacin (LEV) & $0 / 9$ & $0 / 5$ & 2/14 (14.3\%) & $12(85.7 \%)$ & 14 \\
\hline Total (\%) & $1 / 16(6.2 \%)$ & $2 / 40(5 \%)$ & $3 / 56(5.3 \%)$ & $50(89.3)$ & 56 \\
\hline
\end{tabular}

${ }^{a}$ Using the results from the susceptibility testing of the bacteria by Vitek 2 as reference. In case of discrepancies, the results for the susceptibility testing were confirmed by E-test. Data are numbers (with percentages) of bacterial isolates for which the antimicrobial susceptibility testing was concordant/correct or erroneous by PR1 (A) or PR2 (B)

levofloxacin, and amikacin by PR1, and for levofloxacin, ceftazidime, amikacin, and colistin by PR2. In the overall, no significant difference in accuracy was found between PR1 and PR2 for Gram-negative bacteria $(0.2<P<0.5)$.

For Enterobacteriaceae (Table 3), categorical AST agreement was 88.1 by PR1, and 90.7 by PR2. By PR1, there were 26/148 (17.6\%) major errors, and 10/301 (3.3 \%) minor errors; by PR2, there were 6/145 (4.1\%) very major errors, 15/142 (10.6\%) major errors, and 6/ $292(2.1 \%)$ minor errors. All the very major errors were observed for cefotaxime by PR2, whereas an AST agreement of $94.9 \%$ was observed by PR1 for this antibiotic. On the contrary, ceftazidime reached significantly $(p<0.05)$ higher AST agreement by PR2 (93.1\%) than by PR1 (76.7 \%). Of interest, AST agreement for levofloxacin was $100 \%$ by both PR1 and PR2. Comparable AST agreement, below $90 \%$, was observed by PR1 and PR2 with gentamicin and meropenem.

For nonfermenters (Table 4), categorical AST agreement was 86.5 by PR 1 and 89.3 by PR2. By PR 1 there were $3 / 15$ (20 \%) very major errors, 2/37 (5.4\%) major errors, and 2/ 52 (3.8 \%) minor errors; by PR2 there were 1/16 (6.2 \%) very major errors, $2 / 40$ (5\%) major errors, and $3 / 56$ $(5.3 \%)$ minor errors. Of interest, AST agreement for amikacin was $100 \%$ by both PR1 and PR2. AST agreement was $100 \%$ also for colistin by PR2. AST agreement below $90 \%$ was observed by PR1 and PR2 with gentamicin and levofloxacin, and with colistin by PR1.

\section{Direct AST of Gram-positive cocci}

The AST results of 103 Gram-positive cocci were analysed, 76 (73.8 \%) of which were coagulase-negative staphylococci, 17 (16.5\%) S. aureus, 8 (7.8 \%) Enterococcus spp., and $2(1.9 \%)$ streptococci; no profiles were available for $1 S$. epidermidis by PR1, and for 6 isolates (3 S. hominis, 1 S. aureus, 1 Enterococcus faecium, 1 S. pneumoniae) by PR2.

The antimicrobial susceptibility testing of Grampositive cocci (Tables 5 and 6) was performed for a total of 305 and 290 isolate/antimicrobial agent combinations by PR1 and PR2, respectively. Discrepancies in the AST results between the direct and current methods were resolved by Etest, which revealed that the AST was correct by PR1 or PR2, but not by the current method, for 13 (4.3) and 14 (4.8\%) isolate/antimicrobial agent combinations, respectively, all of which regarding coagulase-negative staphylococci. The AST results by PR1 and PR2 and the percent errors are shown in Tables 5 and 6. Complete AST agreement was found for 81 (78.6 \%) isolates by PR1 and 79 (76.7 \%) by PR2, and for all S. aureus strains by PR1. Together, 93.1 and $93.8 \%$ of the isolate/antimicrobial agent combinations showed concordant/correct AST results for Gram-positive cocci by PR1 and PR2, respectively. Of note, AST agreement with linezolid was 99 and $100 \%$, respectively, by PR1 and PR2. No significant difference in accuracy was found between PR1 and PR2 for Gram-positive cocci $(0.5<P<0.9)$.

For staphylococci (Table 5), by PR1 there were $1 / 66$ $(1.5 \%)$ very major errors, $12 / 210$ (5.7 \%) major errors, and 5/276 (1.8 \%) minor errors, and by PR2 there were $3 / 64(4.7 \%)$ very major errors, $11 / 203$ (5.4 \%) major errors, and 3/267 (1.1 \%) minor errors. All the very major errors were observed with cefoxitin by both PR1 (one case) and PR2 (three cases). AST agreement was higher than $90 \%$ for all the antibiotics tested by PR1, and for all but cefoxitin by PR2. Complete AST agreement was observed with linezolid by PR2. 
Table 5 Antimicrobial susceptibility testing of Staphylococcus species from positive blood cultures by PR1 (A) or PR2 (B) ${ }^{a}$

\begin{tabular}{lllll}
\hline Antimicrobial agent & No. of very major errors & No. of major errors & No. of minor errors & AST Agreement \\
\hline A & & & & Total \\
Cefoxitin (CFX) & $1 / 63(1.6 \%)$ & $5 / 29(17.2 \%)$ & $2 / 92(2.2 \%)$ & $84(91.3 \%)$ \\
Linezolid (LZ) & $0 / 3$ & $1 / 89(1.1 \%)$ & $0 / 92$ & $91(98.9 \%)$ \\
Teicoplanin (TEl) & $0 / 0$ & $6 / 92(6.5 \%)$ & $3 / 92(3.3 \%)$ & $83(90.2 \%)$ \\
Total (\%) & $1 / 66(1.5 \%)$ & $12 / 210(5.7 \%)$ & $5 / 276(1.8 \%)$ & $258(93.5 \%)$ \\
B & & & & $79(88.7 \%)$ \\
Cefoxitin (CFX) & $3 / 61(4.7 \%)$ & $4 / 28(14.3 \%)$ & $3 / 89(3.4 \%)$ & $89(100 \%)$ \\
Linezolid (LZ) & $0 / 3$ & $0 / 86$ & $0 / 89$ & $82(92.1 \%)$ \\
Teicoplanin (TEl) & $0 / 0$ & $7 / 89(7.9 \%)$ & $0 / 89$ & 89 \\
Total (\%) & $3 / 64(4.7 \%)$ & $11 / 203(5.4 \%)$ & $3 / 267(1.1 \%)$ & 89 \\
\hline
\end{tabular}

${ }^{a}$ Using the results from the susceptibility testing of the bacteria by Vitek 2 as reference. In case of discrepancies, the results for the susceptibility testing were confirmed by E-test. Data are numbers (with percentages) of bacterial isolates for which the antimicrobial susceptibility testing was concordant/correct or erroneous by PR1 (A) or PR2 (B)

For streptococci/enterococci (Table 6), representing only $9.7 \%$ of all the Gram-positive cocci tested, by PR1 there were $1 / 6(16.7 \%)$ very major errors, and $2 / 23$ (8.7 \%) major errors, and by PR2 there was $1 / 20$ (5 \%) major error. Complete agreement for all isolates was observed with linezolid by both PR1 and PR2, and with ampicillin by PR2. AST agreement below $90 \%$ was obtained with teicoplanin by both PR1 and PR2, and with ampicillin by PR1.

\section{Discussion}

In the last few years, the rapid development of MALDITOF MS technology has allowed rapid bacterial species identification directly from positive BC $[10,11,24-30]$.

In the present study, direct MALDI-TOF analysis was used for rapid identification of bacteria from monomicrobial BC, as previously described [11]. Concordant identification of Gram-negative bacteria and Grampositive cocci by the direct MALDI-TOF method compared to the current method was $97.5 \%$ and $96.1 \%$, respectively, supporting that direct MALDI-TOF can be used efficiently for ID of bacteria in positive BC [11, 27, $29,30]$. However, it should be noted that only score values $\geq 2.000$ are considered as probable species identification. As we observed in a previous study, our data strongly suggest that cut-off values could be lowered down to 1.4 without compromising accuracy [11]. Similar observations have been reported also by other authors $[28,30,31]$. Therefore, the availability of rapid ID results by this method allowed proceeding with the selection of the proper antibiotic panel for direct AST on the basis of those available for this new system. The main finding from the present study is that the proposed method for direct AST might be used to provide clinicians with reliable AST results in a few hours. The AST results of Gram-negative bacteria obtained by the two different protocols, PR1 and PR2, were concordant/correct with the results obtained by the current method for 87.8 and $90.5 \%$ of the isolate/antimicrobial agent combinations, respectively. For Gram-negative

Table 6 Antimicrobial susceptibility testing of streptococci/enterococci from positive blood cultures by PR1 (A) or PR2 (B)

\begin{tabular}{|c|c|c|c|c|c|}
\hline Antimicrobial agent & No. of very major errors & No. of major errors & No. of minor errors & AST Agreement & Total \\
\hline \multicolumn{6}{|l|}{$A$} \\
\hline Ampicillin (AMP) & $1 / 5(20 \%)$ & $0 / 4$ & $0 / 9$ & $8(88.9 \%)$ & 9 \\
\hline Linezolid (LZ) & $0 / 0$ & $0 / 10$ & $0 / 10$ & 10 (100\%) & 10 \\
\hline Teicoplanin (TEI) & $0 / 1$ & $2 / 9(22.2 \%)$ & $0 / 10$ & $8(80 \%)$ & 10 \\
\hline Total (\%) & $1 / 6(16.7 \%)$ & $2 / 23(8.7 \%)$ & $0 / 29$ & $26(89.7 \%)$ & 29 \\
\hline \multicolumn{6}{|l|}{ B } \\
\hline Ampicillin (AMP) & $0 / 3$ & $0 / 4$ & $0 / 7$ & $7(100 \%)$ & 7 \\
\hline Linezolid (LZ) & $0 / 0$ & $0 / 8$ & $0 / 8$ & $8(100 \%)$ & 8 \\
\hline Teicoplanin (TEI) & $0 / 0$ & $1 / 8(12.5 \%)$ & $0 / 8$ & 7 (87.5 \%) & 8 \\
\hline Total (\%) & $0 / 3$ & $1 / 20(5 \%)$ & $0 / 23$ & $22(95.7 \%)$ & 23 \\
\hline
\end{tabular}

${ }^{a}$ Using the results from the susceptibility testing of the bacteria by Vitek 2 as reference. In case of discrepancies, the results for the susceptibility testing were confirmed by E-test. Data are numbers (with percentages) of bacterial isolates for which the antimicrobial susceptibility testing was concordant/correct or erroneous by PR1 (A) or PR2 (B) 
bacteria, levofloxacin showed an agreement in clinical categories $>90 \%$ by both PR1 and PR2, with $<3 \%$ very major errors and $<7 \%$ major and minor errors in combination, thus meeting the selection criteria for an antimicrobial susceptibility testing proposed by Jorgensen [32].

For Enterobacteriaceae, there was $100 \%$ categorical agreement for levofloxacin by both PR1 and PR2, $94.9 \%$ for cefotaxime by PR1 and $93.1 \%$ for ceftazidime by PR2. The lowest categorical agreement was found for ceftazidime by PR1 (significantly lower than by PR2). Unfortunately, meropenem and ceftazidime tests were not available for nonfermenters. Although the number of nonfermenters tested was low, $100 \%$ categorical agreement was observed with amikacin by PR1 and PR2, and with colistin by PR2. Altogether, no significant difference in accuracy was found for Gram-negative bacteria between PR1 and PR2.

The AST results of Gram-positive cocci by PR1 and PR2 were concordant/correct with the results obtained by the current method for 93.1 and $93.8 \%$ of the isolate/ antibiotic combinations, respectively. Cefoxitin (91.3\%) and linezolid $(99.0 \%)$ by PR1, and linezolid (100 \%) and teicoplanin $(91.8 \%)$ by PR2 showed an agreement in clinical categories $>90 \%$, with linezolid meeting the selection criteria proposed by Jorgensen, both by PR1 and PR2. With the only exception of cefoxitin $(88.7 \%)$ by PR2, there was a categorical agreement $>90 \%$ for all the antimicrobial agents tested on staphylococci by both PR1 and PR2. Optimal results were obtained for $S$. aureus isolates by PR1, with 17/17 isolates showing complete AST agreement with the current method. Although the number of streptococci/enterococci tested was low, $100 \%$ categorical agreement was observed with linezolid, by PR1 and PR2, and ampicillin by PR2. In the overall, no significant difference in accuracy was found between PR1 and PR2 for Gram-positive cocci. The time required by PR1 from blood culture positivity to AST results is about $4 \mathrm{~h}$, including about $30 \mathrm{~min}$ to prepare the bacterial suspension and obtaining ID results, and a three-hour incubation for most of the tested antibiotics. For antibiotics requiring a five-hour incubation, $6 \mathrm{~h}$ are needed to provide AST results by PR1. The time required by PR2 from blood culture positivity to AST results is more variable, depending on the time required to reach the $0.5 \mathrm{McF}$ suspension (1-4 h) and the whole procedure may take up to $9 \mathrm{~h}$. On the bases of these observations, PR1 is to be preferred to PR2.

\section{Conclusions}

The most relevant conclusion from the present study is that the proposed method allows to yield rapid and reliable AST results, which often is of vital importance to either confirm or streamline antibiotic therapy for patients suffering from BSI. In addition, the earlier administration of appropriate antimicrobial therapy will also reduce the emergence of antimicrobial-resistant strains. The results were particularly favorable for Gram-positive cocci, which often yield less accurate results than Gram-negative bacteria when tested directly from positive BC. In particular, $100 \%$ categorical agreement was found with levofloxacin for Enterobacteriaceae by both PR1 and PR2, and 99.0 and $100 \%$ categorical agreement was observed with linezolid for Gram-positive cocci by PR1 and PR2, respectively. A limitation of this study is that times to result were not accurately measured. Further studies involving a higher number of blood samples and antibiotics are needed to fully evaluate the potential of this new method. Important advancement in patient care in terms of clinical and financial benefits is to be expected by its implementation in clinical microbiological laboratories.

\section{Additional file}

Additional file 1: Table S1. Number of susceptible, intermediate and resistant isolates for each species/antimicrobial agent combination according to the current method: Enterobacteriaceae (A), Gram-negative nonfermenters (B), staphylococci (C), and streptococci-enterococci (D). (DOCX $17 \mathrm{~kb})$

\section{Abbreviations \\ AST, antimicrobial susceptibility testing; BC, blood cultures; BSI, bloodstream infections; ID, identification; MALDI-TOF, matrix-assisted laser desorption/ ionization time-of-flight; PR1, protocol 1; PR2, protocol 2; MIC, minimum inhibitory concentration}

\section{Acknowledgements}

The Authors pay tribute to the late Prof. Mario Campa for his long standing, inspiration and support.

Part of the study materials were kindly provided by Alifax SpA (PD, Italy).

\section{Funding}

This study was supported by a research grant from the Italian "Ministero dell'Istruzione, dell'Università e della Ricerca", contract n²012WJSX8K_005.

\section{Availability of data and materials}

The datasets analyzed during the current study are available from the corresponding author on request.

\section{Authors' contributions}

Conceived and designed the experiments: SB, AL. Performed the experiments: VB, PM. Analyzed the data: SB, VB, WF, AL. Wrote the manuscript: WF, EG, AL. All authors read and approved the final manuscript.

\section{Competing interests}

The authors declare that they have no competing interests.

\section{Consent for publication}

Not applicable.

\section{Ethics approval and consent to participate}

The study was notified to and approved by the local ethical committee, Comitato Etico di Area Vasta Nord-Ovest, University of Pisa, and conducted in full accordance with the principles of the Declaration of Helsinki. Samples were taken as part of the standard patient care and used anonymously. For this type of study no written informed consent was necessary since this study was performed using otherwise wasted biological samples, and personally identifiable information was removed and destroyed. 


\section{Author details}

${ }^{1}$ Azienda Ospedaliero-Universitaria Pisana, Pisa, Italy. ${ }^{2}$ Dipartimento di Ricerca Traslazionale e delle Nuove Tecnologie in Medicina e Chirurgia, Università di Pisa, Pisa, Italy.

Received: 14 April 2016 Accepted: 4 August 2016

Published online: 12 August 2016

\section{References}

1. Doern GV, Vautour R, Gaudet M, Levy B. Clinical impact of rapid in vitro susceptibility testing and bacterial identification. J Clin Microbiol. 1994;32:1757-62.

2. Barenfanger J, Drake C, Kacich G. Clinical and financial benefits of rapid bacterial identification and antimicrobial susceptibility testing. J Clin Microbiol. 1999;37:1415-8.

3. Kerremans JJ, Verboom P, Stijnen T, Hakkaart-van Roijen L, Goessens W, Verbrugh $\mathrm{HA}$, et al. Rapid identification and antimicrobial susceptibility testing reduce antibiotic use and accelerate pathogen-directed antibiotic use. J Antimicrob Chemother. 2008;61:428-35.

4. Vlek AL, Bonten MJ, Boel CH. Direct matrix-assisted laser desorption ionization time-of-flight mass spectrometry improves appropriateness of antibiotic treatment of bacteremia. PLoS One. 2012;7:e32589.

5. Kollef MH, Ward S, Sherman G, Prentice D, Schaiff R, Huey W, et al. Inadequate treatment of nosocomial infections is associated with certain empiric antibiotic choices. Crit Care Med. 2000;28:3456-64.

6. Parcell BJ, Orange GV. PNA-FISH assays for early targeted bacteraemia treatment. J Microbiol Methods. 2013;95:253-5.

7. Pletz MW, Wellinghausen $N$, Welte T. Will polymerase chain reaction (PCR)based diagnostics improve outcome in septic patients? A clinical view Intensive Care Med. 2011;37:1069-76.

8. Lupetti A, Barnini S, Dodi C, Menconi M, Favre C, Giagnoni M, et al. New rapid methods cannot replace the current method to diagnose bloodstream infections. J Med Microbiol. 2014;63:767-9.

9. Wieser A, Schneider L, Jung J, Schubert S. MALDI-TOF MS in microbiological diagnostics-identification of microorganisms and beyond (mini review). Appl Microbiol Biotechnol. 2012;93:965-74.

10. Clerc O, Prod'hom G, Vogne C, Bizzini A, Calandra T, Greub G. Impact of matrix-assisted laser desorption ionization time-of-flight mass spectrometry on the clinical management of patients with gram-negative bacteremia: a prospective observational study. Clin Infect Dis. 2013;56:1101-7.

11. Barnini S, Ghelardi E, Brucculeri V, Morici P, Lupetti A. Rapid and reliable identification of gram-negative bacteria and gram-positive cocci by deposition of bacteria harvested from blood cultures onto the MALDI-TOF plate. BMC Microbiol. 2015;15:124.

12. Hansen DS, Jensen AG, Nørskov-Lauritsen N, Skov R, Bruun B. Direct identification and susceptibility testing of enteric bacilli from positive blood cultures using VITEK (GNI+/GNS-GA). Clin Microbiol Infect. 2002;8:38-44.

13. Ling TK, Liu ZK, Cheng AF. Evaluation of the VITEK 2 system for rapid direct identification and susceptibility testing of gram-negative bacilli from positive blood cultures. J Clin Microbiol. 2003:41:4705-7.

14. Bruins MJ, Bloembergen P, Ruijs GJ, Wolfhagen MJ. Identification and susceptibility testing of Enterobacteriaceae and Pseudomonas aeruginosa by direct inoculation from positive BACTEC blood culture bottles into Vitek 2. J Clin Microbiol. 2004;42:7-11.

15. Funke G, Funke-Kissling P. Use of the BD PHOENIX automated microbiology system for direct identification and susceptibility testing of gram-negative rods from positive blood cultures in a three-phase trial. J Clin Microbiol. 2004:42:1466-70.

16. Lupetti A, Barnini S, Castagna B, Nibbering PH, Campa M. Rapid identification and antimicrobial susceptibility testing of gram-positive cocci in blood cultures by direct inoculation into the BD phoenix system. Clin Microbiol Infect. 2010;16:986-91.

17. Beuving J, van der Donk CF, Linssen CF, Wolffs PF, Verbon A. Evaluation of direct inoculation of the BD PHOENIX system from positive BACTEC blood cultures for both gram-positive cocci and gram-negative rods. BMC Microbiol. 2011;11:156.

18. Lupetti A, Barnini S, Morici P, Ghelardi E, Nibbering PH, Campa M. Saponin promotes rapid identification and antimicrobial susceptibility profiling of gram-positive and gram-negative bacteria in blood cultures with the Vitek 2 system. Eur J Clin Microbiol Infect Dis. 2013;32:493-502.
19. Florio W, Barnini S, Morici P, Lupetti A. Direct inoculation of positive blood cultures using the phoenix system for antimicrobial susceptibility testing of both gram-positive and gram-negative bacteria. J Med Microbiol. 2015:64:582-5.

20. Gherardi G, Angeletti S, Panitti M, Pompilio A, Di Bonaventura G, Crea F, et al. Comparative evaluation of the Vitek-2 compact and phoenix systems for rapid identification and antibiotic susceptibility testing directly from blood cultures of gram-negative and gram-positive isolates. Diagn Microbiol Infect Dis. 2012;72:20-31.

21. Wimmer JL, Long SW, Cernoch P, Land GA, Davis JR, Musser JM, et al. Strategy for rapid identification and antibiotic susceptibility testing of gram-negative bacteria directly recovered from positive blood cultures using the bruker MALDI biotyper and the BD phoenix system. J Clin Microbiol. 2012;50:2452-4.

22. Lahanas S, Stathopoulos G, Chan RC, van Hal SJ. Evaluation of the Alfred 60/ AST device as a screening test for urinary tract infections. J Clin Microbiol. 2013;51:3406-8.

23. European Committee for Antimicrobial Susceptibility Testing (EUCAST) of the European Society of Clinical Microbiology and Infectious Diseases (ESCMID). EUCAST Definitive Document E. DEF 2.1 August 2000. Determination of antimicrobial susceptibility test breakpoints. Clin Microbiol Infect. 2000;6:570-2.

24. La Scola B, Raoult D. Direct identification of bacteria in positive blood culture bottles by matrix-assisted laser desorption ionisation time-of-flight mass spectrometry. PLoS One. 2009;: :e8041.

25. Machen A, Drake T, Wang YF. Same day identification and full panel antimicrobial susceptibility testing of bacteria from positive blood culture bottles made possible by a combined lysis-filtration method with MALDI-TOF VITEK mass spectrometry and the VITEK2 system. PLoS One. 2014;9:e87870.

26. Stevenson LG, Drake SK, Murray PR. Rapid identification of bacteria in positive blood culture broths by matrix-assisted laser desorption ionization-time of flight mass spectrometry. J Clin Microbiol. 2010;48:444-7.

27. Christner M, Rohde H, Wolters M, Sobottka I, Wegscheider K, Aepfelbacher M. Rapid identification of bacteria from positive blood culture bottles by use of matrix-assisted laser desorption-ionization time of flight mass spectrometry fingerprinting. J Clin Microbiol. 2010;48:1584-91.

28. Lagacé-Wiens PR, Adam HJ, Karlowsky JA, Nichol KA, Pang PF, Guenther J, et al. Identification of blood culture isolates directly from positive blood cultures by use of matrix-assisted laser desorption ionization-time of flight mass spectrometry and a commercial extraction system: analysis of performance, cost, and turnaround time. J Clin Microbiol. 2012;50:3324-8.

29. Fothergill A, Kasinathan V, Hyman J, Walsh J, Drake T, Wang YF. Rapid identification of bacteria and yeasts from positive-blood-culture bottles by using a lysis-filtration method and matrix-assisted laser desorption ionization-time of flight mass spectrum analysis with the SARAMIS database. J Clin Microbiol. 2013;51:805-9.

30. Buchan BW, Riebe KM, Ledeboer NA. Comparison of the MALDI Biotyper system using Sepsityper specimen processing to routine microbiological methods for identification of bacteria from positive blood culture bottles. J Clin Microbiol. 2012;50:346-52.

31. Saffert RT, Cunningham SA, Mandrekar J, Patel R. Comparison of three preparatory methods for detection of bacteremia by MALDI-TOF mass spectrometry. Diagn Microbiol Infect Dis. 2012;73:21-6.

32. Jorgensen $\mathrm{JH}$. Selection criteria for an antimicrobial susceptibility testing system. J Clin Microbiol. 1993;31:2841-4.

\section{Submit your next manuscript to BioMed Central} and we will help you at every step:

- We accept pre-submission inquiries

- Our selector tool helps you to find the most relevant journal

- We provide round the clock customer support

- Convenient online submission

- Thorough peer review

- Inclusion in PubMed and all major indexing services

- Maximum visibility for your research

Submit your manuscript at www.biomedcentral.com/submit 